\title{
A METEOROLOGICAL RISK ASSESSMENT METHOD FOR POWER LINES BASED ON GIS AND MULTI-SENSOR INTEGRATION
}

\author{
Zhiyong $\operatorname{Lin}^{\mathrm{a}}$, Zhimin $\mathrm{Xu}^{\mathrm{a}}{ }^{\mathrm{a}}$, * \\ ${ }^{\text {a }}$ School of Remote Sensing and Information Engineering, Wuhan University, Wuhan, China - zhylin@whu.edu.cn \\ ${ }^{\mathrm{b}}$ Changjiang Spatial Information Technology Engineering Co., Ltd, Wuhan, China - xuzhimin@whu.edu.cn
}

\section{Commission VIII, WG VII/1}

KEY WORDS: Risk assessment, Multi-sensor, Power lines, Analytic hierarchy process

\begin{abstract}
:
Power lines, exposed in the natural environment, are vulnerable to various kinds of meteorological factors. Traditional research mainly deals with the influence of a single meteorological condition on the power line, which lacks of comprehensive effects evaluation and analysis of the multiple meteorological factors. In this paper, we use multiple meteorological monitoring data obtained by multi-sensors to implement the meteorological risk assessment and early warning of power lines. Firstly, we generate meteorological raster map from discrete meteorological monitoring data using spatial interpolation. Secondly, the expert scoring based analytic hierarchy process is used to compute the power line risk index of all kinds of meteorological conditions and establish the mathematical model of meteorological risk. By adopting this model in raster calculator of ArcGIS, we will have a raster map showing overall meteorological risks for power line. Finally, by overlaying the power line buffer layer to that raster map, we will get to know the exact risk index around a certain part of power line, which will provide significant guidance for power line risk management. In the experiment, based on five kinds of observation data gathered from meteorological stations in Guizhou Province of China, including wind, lightning, rain, ice, temperature, we carry on the meteorological risk analysis for the real power lines, and experimental results have proved the feasibility and validity of our proposed method.
\end{abstract}

\section{INTRODUCTION}

Power lines are important infrastructures for national electric power distribution and transmission. In order to maintain power supply stability and safeguard the national economic development, strengthening the power line safe operation maintenance is particularly important. Power lines, exposed in the natural environment, are not only affected by normal mechanical load and power load, but also vulnerable to various kinds of meteorological factors, such as wind, rain, ice, snow, ray, high temperature, etc. These factors will lead to aging, oxidation and corrosion of electrical components on the power line. If not take timely prevention measures, all kinds of power line failures will occur, like trip, break or short circuit (Aggarwal et al., 2000). The operation of power lines is influenced by various meteorological factors. Hence, multisource meteorological information needs to be integrated to implement comprehensive analysis in the process of power line inspection and equipment health monitoring.

In recent years, multi-sensor information fusion technology has been widely used in such fields as military, marine, earthquake, construction, due to its characteristics of fault tolerance, complementarity, real-time and economy. Using multi-sensor information fusion technology, the overall meteorological environment surrounding power lines can be perceived, which is contributed to improve the efficiency and accuracy of power line meteorological risk monitoring. That will reinforce the government's capability of power line disaster management and emergency response, and guarantee the safety and reliability of power lines for the economic and social service. Therefore, in this paper, a meteorological risk assessment method for power lines, using multi-sensor fusion technology and GIS spatial analysis, is proposed. And three key points concerning the method are specifically discussed, including the framework of meteorological risk, the weight design of meteorological factors and the extraction of meteorological risk value. Furthermore, using the discrete meteorological point data obtained by five kinds of meteorological sensors distributed in Guizhou Province in southern China, experimental studies are carried out to implement the power line meteorological risk analysis and early-warning, and the results of which are validated by sensitivity analysis and correlation analysis.

The rest of the paper is organized as follows. Section 2 briefly summarizes the related works in meteorological risk modelling and GIS applications in power line management. Section 3 introduces the methods and approaches of meteorological risk assessment. Section 4 presents a case study for power lines in Guizhou Province and analyses the experimental results. Section 5 concludes the paper.

\section{RELATED WORKS}

Power lines in the natural environment will be affected by various meteorological factors, these factors will bring hidden danger to the secure and stable operation of the power line. Academic circles have done lots of research against how meteorological factors, like wind and rain, snow and ice, lightning and high temperature, affect the safe operation of the

* Corresponding author 
power lines. Bai and Li thoroughly studied dynamic response of overhead transmission lines to oscillation caused by wind or rainfall loads using three degree of freedom model (Bai and $\mathrm{Li}$, 2009). Kikuchi and Matsuzaki et al. figured out the aerodynamic characteristic of grooved round transmission wires in wind-tunnel experiments with a factor of rainfall attached (Kikuchi et al., 2003). Savadjiev et al. performed statistical analysis of meteorological data for icing and ice shedding on overhead power-line conductors and explored the correlations between hourly icing rate and the variations of the multiple meteorological variables (Savadjiev and Farzaneh, 2004). Jakse et al. explored the static and dynamic snow-shedding effects on $110 \mathrm{kv}$ overhead power lines in Slovenia using numerical simulations (Jakše et al., 2001).

By comparison and analysis of relative literature, we can see that past researches mainly consider single meteorological conditions and focus on the mathematical model to meet the requirement of power line reliability assessment. But there are few researches on risk analysis and early warning which consider multiple meteorological factors as a whole. GIS is a widely used tool with powerful data storage, management and sharing ability and rich spatial analysis ability, which can effectively integrate multi-source sensor data, provide spatial data support for all kinds of disaster management and decision support analysis and thus significantly improve the work efficiency and management level (Qi and Altinakar, 2011; Tang and Wen, 2009; Zerger and Smith, 2003). However, current GIS applications in power line management mainly satisfy the need for basic functions in the power line industry, such as power line layout, minimum cost path analysis, evaluation of environmental impact factors, power line measurement and calculation, three dimensional display and analysis, thematic map and statistics output (Bagli et al., 2011; Yang et al., 2010). Some higher level of applications can implement power information sharing and unified management, but abilities in risk analysis, early warning and decision making are very limited (Haihong et al., 2013; Jianli et al., 2003). So the advantages of GIS in data integration have not been fully realized.

To sum up, the traditional power line meteorological risk researches focus on only one meteorological factor, which is difficult to assess overall meteorological risk. Existing power line management system implements power line information management, sharing and monitoring, but lack of research on power line meteorological risk analysis and decision-making. Therefore, based on multiple meteorological data acquired by multi-sensor technology, this paper uses analytic hierarchy analysis and GIS spatial analysis to implement power line meteorological risk modelling and decision-making, which might be able to make up for the deficiency of the traditional method.

\section{METHODS AND APPROACHES}

\subsection{Framework of the meteorological risk for power lines}

Natural disaster risk is defined as the hazard-related potential adverse effects and total economic loss expected in a region as a composite function of hazard, exposure, vulnerability, and management ability (Davidson and Lambert, 2001). But definitions of risk in research vary with their applications. Their bases lie in the common sense understanding of the concept of risk (Wang et al., 2011). Usually, the risk index value is associated with the probability and the severity of the hazard. In this paper, the power line meteorological risk index (PLMRI) is represented using the following function:

$$
\text { PLMRI }=\sum_{i=1}^{n} \omega_{i} v_{i}
$$

Here, $v$ is the normalized value of every different selected meteorological factors, $\mathrm{n}$ is the number of the meteorological factors, $\omega$ is the weight value referring to a certain meteorological factor and the sum of every different $\omega$ is one.

There are two different normalization methods, according to the different relationship between the meteorological value and PLMRI value; for example, positive correlation or negative correlation (Liu et al., 2012; Wang et al., 2011). As to the positive indicator, the minimum method equation (2) was used, whereas the maximum method equation (3) was applied to the negative indicator.

$$
\begin{aligned}
& v=\frac{v_{\max }-v}{v_{\max }-v_{\min }} \\
& v=\frac{v-v_{\min }}{v_{\max }-v_{\min }}
\end{aligned}
$$

Our framework first allows the user to generate a raster map for each kind of meteorological point data using different spatial interpolation method and normalization method. In every raster map, each cell has a risk value $v$ that represents how much potential risk the meteorological factor will have on the power lines. After that, PLMRI raster map with the same cell size will be generated. The PLMRI value of each cell is the weighted sum of every risk value that belongs to the cell in every meteorological raster map with same location. The weight of each meteorological factor is designed using analytic hierarchy process which starts from construction of relative importance matrix. Finally the PLMRI along the power line can be extracted by buffer and overlay analysis between line feature and raster map and eventually be output as statistical diagram. The framework of our research is presented as follow.

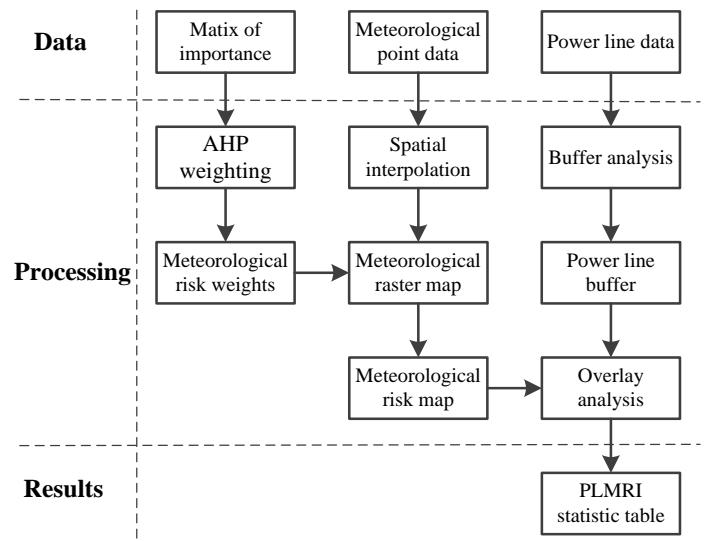

Figure 1 Framework of the meteorological risk for power lines

\subsection{Selection of indicators}

This paper selects five kinds of meteorological factors to model the potential risk. They are wind, rain, lightning, icing and high temperature, which have caused different hazards to power lines as many news report (shown in Figure 2). And the reasons are given below. As the five selected meteorological factors are all 
positively correlated with the meteorological risk value, so equation (3) is chosen as the normalization method for all meteorological data.

1) Wind. Under the effect of wind, the suspension insulator strings attached to power line tower will tilt, making the air gap between the power line and the power line tower smaller, which will easily result in interphase short circuit and wire burnout accident (Abdel-Gawad and Zoklot, 2006). The unit of the wind in this paper is meters per second.

2) Rains. Drizzle will cause pollution flashover and discharge of insulator discharge, which will result in damage to the insulator . And heavy rainfall like downpour will make surging river, flash floods and landslides, causing power line tower fall and power line break. The unit of the wind in this paper is millimetre per hour.

3) Lightning. The most part of the overhead distribution lines are exposed in the air, so they are apt to be damaged by lightning and cause lightning over voltages, which will interrupt power supply and affect users' production and lives. In this paper, the intensity of lightning is measured by the density of lightning stroke in a kilometre grid.

4) Icing. When power lines are covered with heavy ice accretion, it may break up. And when ice accretion falls off, it will make the power line sway and increase the potential risk of flashover accident (Farzaneh and Savadjiev, 2005). Like lightning data above, the ice data is based on the historical statistics which have been classified into seven levels.

5) Temperature. Power line will expand when heated and contract when cooled and the tension will also change with the temperature of high or low. As the temperature is high in summer, the power line will sag, which will cause discharge in intersection and grounding short circuit accident (Thompson and Pilgrim, 2000). The unit of the temperature in this paper is degree centigrade.

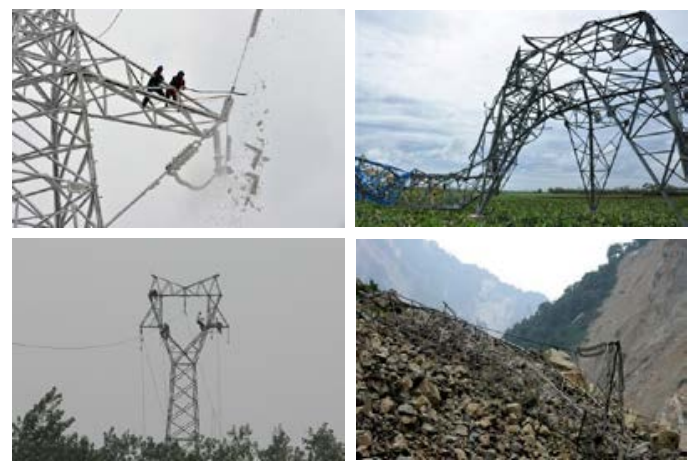

Figure 2 Power line damages brought by extreme weather

\subsection{Spatial interpolation of meteorological data}

According to the first law of geography, everything is related to everything else, but near things are more related than distant things (Tobler, 1970). Spatial interpolation, based on the first law of geography, is a collection of methods that allow users to estimate values for locations where no sample points have been taken and also to assess the uncertainty of these estimates. These functions are important in many decision-making processes, as it is impossible in practice to take samples at every location in an area of interest. There are many interpolation methods exist, such as polynomial method, inverse distance weighted, radial basis functions, diffusion kernel, kernel smoothing and kriging method. Some are quite flexible and others are more restrictive. Different interpolation method requires that the data meet specific conditions. In this paper, the meteorological data is collected by multi-sensors in weather station distributed in different places and stored in shapefile as point data. So it is necessary to use different spatial interpolation method to estimate different meteorological value for locations where no sample points exist.

For wind, rains and temperature, these three kinds of meteorological phenomenon is spatially continuously distributed in nature. For example, the intensity of wind is continuously changing and is becoming smaller and smaller from the eye of the wind to the edge. Thus, these three kinds of meteorological data are suitable for inverse distance weighted (IDW) method. This method uses the measured values surrounding the prediction location to predict a value for any unsampled location, based on the assumption that things that are close to one another are more alike than those that are farther apart. Given a set of weather station location points $\left(x_{i}, y_{i}\right)$ and $z_{i}$ is the meteorological value, where $i \in(1,2, \cdots n)$. Then the meteorological value $Z$ at any other position $(x, y)$ can be calculated through the following equations.

$$
\left\{\begin{array}{c}
z=\frac{\sum_{i=1}^{n} z_{i} / d_{i}^{2}}{\sum_{i=1}^{n} 1 / d_{i}^{2}} \\
d_{i}^{2}=\left(x-x_{i}\right)^{2}+\left(y-y_{i}\right)^{2}
\end{array}\right.
$$

But for lightning and icing, these two meteorological phenomena are spatially discretely distributed in nature. That is to say, an obvious boundary exists between those places with lightning or icing and those without. So in the circumstances, the Thiessen polygon method is a good choice. The Thiessen polygon method is first used in field of climatology, and it is a partitioning of a plane into regions by taking pairs of points that are close together and drawing a line that is equidistant between them and perpendicular to the line connecting them. Any specific region has only one point as centre, which is closer to any point inside the plane than any other region centre. As is shown in Figure 3, supposed that the intensity data of lightning and icing is collected in different weather station distributed as (a), by applying Thiessen polygon based spatial subdivision, as shown in (b), every weather station will dominates an area of region, in which every specific location holds the same intensity of lightning and icing.

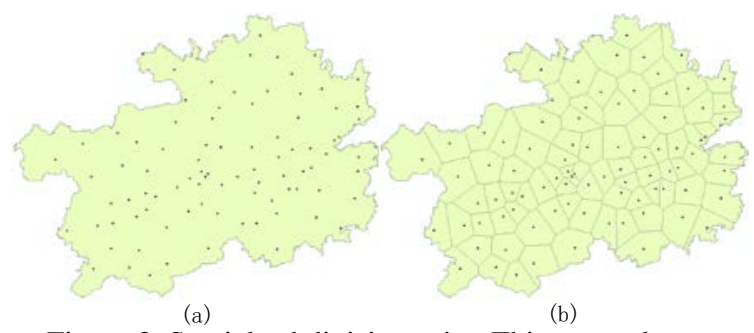

Figure 3 Spatial subdivision using Thiessen polygon 


\subsection{Indicator weights and consistency check}

After the selection of indicators, to assign weights for each indicator is the precondition of PLMRI value calculation according to equation (1). There are many approaches to find weights, including conjunctive/disjunctive approaches, Analytical Hierarchy Process (AHP), the Hasse-Diagramm technique, Outranking approach and Delphi approach. Among these methods, AHP is one of the most widely used multicriteria decision-making tools (Ishizaka and Labib, 2011). AHP provides an effective quantitative decision making tool to deal with complex and unstructured problems. AHP allows a better, easier, and more efficient framework for the identification of selection criteria, calculating their weights and analysis.

The ability to handle both qualitative as well as quantitative data makes AHP an ideal methodology for some prioritization problems by considering different criteria. The process of AHP can be summarized in four steps: construct the decision hierarchy; determine the relative importance of attributes and sub-attributes; evaluate each alternative and calculate its overall weight in regard to each attribute, and check the consistency of the subjective evaluations (Schoenherr et al., 2008). This paper selects five kinds of meteorological factors, thus the decision hierarchy is a one-layer structure as shown in Figure 4.

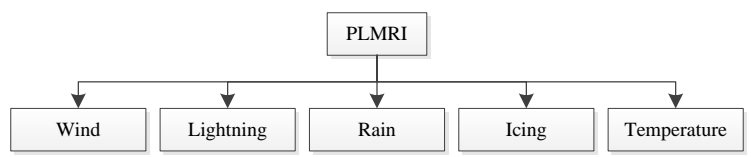

Figure 4 The decision hierarchy for PLMRI

1) Evaluation matrix. Let $v=\{v \mid i=1,2 \cdots n\}$ be a set of meteorological factors. The evaluation matrix $A$ is the result of the pairwise comparison on $\mathrm{n}$ criteria, in which every element $a_{i j}$ is the relative importance between the $\mathrm{i}$-th and $\mathrm{j}$-th criteria using 1-9 relative importance scales method. The nine points are chosen because psychologists conclude that nine objects are the most that an individual can simultaneously compare and consistently rank. Each point indicates the intensity of relative importance and definition of each point is shown in Table 1. For example in equation (5), $a_{12}=3$ means that the first meteorological factor is moderately more important than the second meteorological factor in bringing potential risks to power lines.

$$
A=\left[\begin{array}{cccc}
a_{11} & a_{12} & \cdots & a_{1 n} \\
a_{21} & a_{22} & \cdots & a_{2 n} \\
\vdots & \vdots & \ddots & \vdots \\
a_{n 1} & a_{n 2} & \cdots & a_{n n}
\end{array}\right] a_{i i}=1, a_{i j}=\frac{1}{a_{j i}}
$$

\begin{tabular}{c|c}
\hline Intensity of importance & Definition \\
\hline 1 & Equally important \\
3 & Moderately more important \\
5 & Strongly more important \\
7 & Very strong more important \\
9 & Extremely more important \\
$2,4,6,8$ & Intermediate values \\
\hline
\end{tabular}

Table 1 Nine-point intensity of importance scale
2) Indicator weights calculation. In the mathematical process of AHP, the indicator weights are given by the right eigenvector corresponding to the largest eigenvalue of the evaluation matrix A. In this paper we use square root method to find the eigenvector and the largest eigenvalue of matrix A. First, calculate the product of every matrix elements of each row.

$$
M_{i}=\prod_{j=1}^{n} a_{i j}
$$

Then calculate the n times squaring-roots $\bar{W}_{i}$ of $M_{i}$.

$$
\overline{W_{i}}=\sqrt[n]{\prod_{j=1}^{n} a_{i j}}
$$

Then normalize the vector $\bar{W}=\left(\bar{W}_{1}, \bar{W}_{2}, \cdots \bar{W}_{n}\right)^{T}$. We will get the eigenvector $W=\left(W_{1}, W_{2}, \cdots W_{n}\right)^{T}$, which is the weight value of each indicator.

$$
W_{i}=\frac{\overline{W_{i}}}{\sum_{k=1}^{n} \overline{W_{k}}}
$$

Finally, calculate the largest eigenvalue $\lambda_{\max }$ of matrix A.

$$
\begin{array}{r}
\lambda_{\max }=\sum_{i=1}^{n} \frac{(A W)_{i}}{n W_{i}}=\frac{1}{n}\left(\frac{\sum_{j=1}^{n} a_{1, j} W_{j}}{W_{1}}+\right. \\
\left.\frac{\sum_{j=1}^{n} a_{2 j} W_{j}}{W_{2}}+\cdots+\frac{\sum_{j=1}^{n} a_{n j} W_{j}}{W_{n}}\right)
\end{array}
$$

3) Consistency check. The quality of the output of the AHP is strictly related to the consistency of the pairwise comparison judgments. The consistency is defined by the relation between the entries of $A: a_{i j} * a_{j k}=a_{i k}$. The consistency index $\mathrm{CI}$ is given by equation (10).

$$
C I=\frac{\lambda_{\max }-n}{n-1}
$$

The final consistency ratio CR $=\mathrm{CI} / \mathrm{RI}$, usage of which lets the user to conclude whether the evaluations are sufficiently consistent, is calculated as the ratio of the $\mathrm{CI}$ and the random index RI. When the CR is no more than $10 \%$, we think that the judgment matrix has a satisfactory consistency. Otherwise, adjustment of the judgment matrix is needed.

\subsection{Overlay and buffer analysis for risk assessment}

After the result of indicator weights has passed the consistency check, the PLMRI raster map can be generated based on five kinds of meteorological raster map using equation (1) and ArcGIS raster calculator. In this process, the raster size of every meteorological raster map should keep the same in order to make sure that: (a) every raster grid has a PLMRI value and (b) all raster grids exactly cover the whole geographic area. 


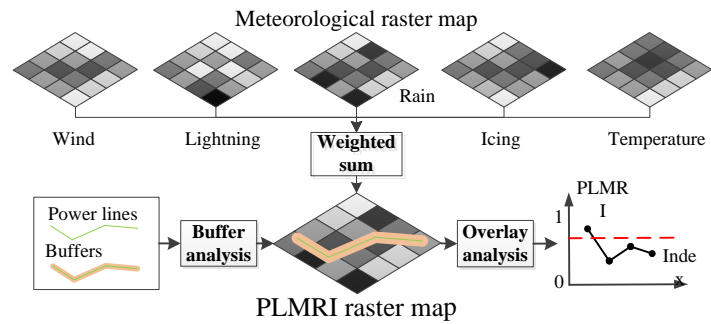

Figure 5 Workflow of PLMRI value extraction

Then centred with the power lines, to generate a buffer polygon section by section with a specific radius and overlay all buffer polygons on the PLMRI raster map to extract the maximum PLMRI value inside each buffer polygon. The whole workflow is shown in Figure 5. That maximum PLMRI value is the quantitative evaluation of the overall meteorological risk that faced with each power line section. When the PLMRI value exceeds a certain threshold, system starts the alarm program to call the power lines manager for special attention or maintenance.

\section{CASE STUDIES}

Guizhou Province is located in the Yun-Gui Plateau of China. With complex meteorological and geological conditions, it is vulnerable to climate hazard and owns high frequency of natural disasters (such as low temperature, freezing, storms, floods, fires, etc.). When a large scale natural disaster occurs, the power grid usually suffers equipment failure, broken line and disconnection accident. As the disaster situation is complex, the disaster assessment and emergency repair work is difficult to carry out timely and effectively. Therefore, this paper chooses Guizhou province as the experimental area, and presents the meteorological risk modeling and analysis based on the multi-sensor meteorological data from 87 weather stations (as shown in Figure 3) in Guizhou province in February 24, 2015.

The major impediment to studying power lines meteorological risk in the study area was the unavailability of GIS data in a digital format. Therefore, in this study, a number of dataset were generated. The boundary data of Guizhou Province are obtained from the National Foundation Geological Information System. The wind speed data, lightning density data, precipitation data, icing intensity data and temperature data were collected from the Meteorological Bureau of Guizhou Province. In the present study, a cell size of $0.018 * 0.018$ radians square was considered as the basic operational unit for analysis.

\subsection{The interpolated raster map of meteorological data}

The three kinds of meteorological data gathered by different meteorological sensors in weather stations are in the form of discrete point data except that the original data of ice and lightning density are raster dataset got from Meteorological Bureau of Guizhou Province. Using inverse distance weighted method, the three kinds of interpolated meteorological raster maps are generated as shown in Figure 6. The reference system of the raster maps is WGS1984 and the raster unit length is 0.018 radians.

The wind speed ranges from 0 to 9 meters per second, the lightning density ranges from 1 to 13.5 times per kilometres square, the rain fall ranges from $0 \mathrm{~mm}$ to $3 \mathrm{~mm}$, the icing intensity ranges from level 1 to level 7 , and the temperature ranges from 16 to 24 degree centigrade. It can be seen from the Figure 6 that the spatial distribution of different kinds of meteorological data are obviously different. Areas with higher wind speed and lightning density are mainly in the west, while areas with more rain falls in the east and areas with higher temperature in the south. So, to get reasonable meteorological risk assessment results, considering only one meteorological factor is one-sided. It is necessary to integrate all the meteorological factors that harm the safe operation of power lines most.

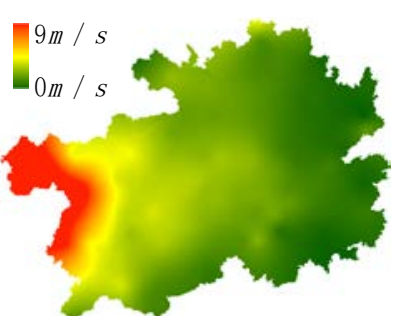

(a) Wind

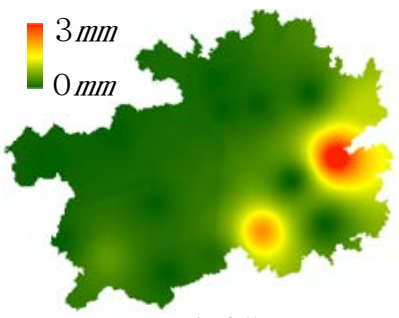

(c) Rain falls

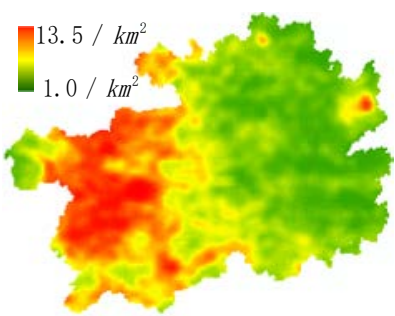

(b) Lightning Density

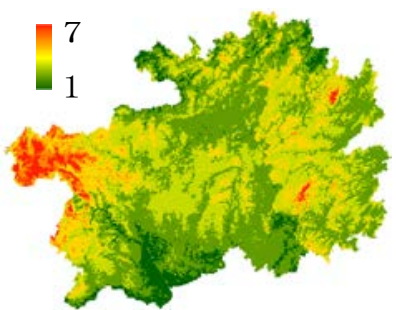

(d) Icing Intensity

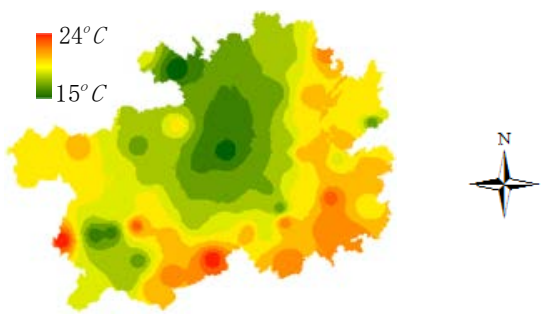

(e) Temperature

Figure 6 Interpolated meteorological raster maps

\subsection{The PLMRI raster map}

According to the conceptual framework, five indicators were selected to represent and quantitatively calculate power line meteorological risk, and the weights were computed by applying AHP method. Three experts in the meteorology and four experts in the energy and utility subjects were selected. Through the issuance of questionnaires, and sort out the evaluation opinions of experts, comparisons are made on a scale of 1-9 (Table 1). This scale was originally chosen to support comparisons within a limited range but with sufficient sensitivity. These pairwise comparisons yield a reciprocal n-by$\mathrm{n}$ matrix A. Weights were obtained by analysis matrix A according to Section 3.4. The consistency is tested as shown in Table 2. The final consistency ratio is $0.075(<0.1)$, so the results pass the test of consistency, which means that the proposed judgment matrix has a satisfactory consistency. The indicators are in the leftmost column, which are wind, lightning, rain, icing and temperature from up to bottom, and the weights of indicators are in the rightmost column (Table 2).

\begin{tabular}{l|llllll}
\hline$A$ & $v_{W}$ & $v_{1}$ & $v_{r}$ & $v_{i}$ & $v_{t}$ & $W_{i}$ \\
\hline
\end{tabular}




\begin{tabular}{c|cccccc}
\hline$v_{W}$ & 1 & 3 & 2 & 2 & 2 & 0.325 \\
$v_{I}$ & $1 / 3$ & 1 & $1 / 3$ & $1 / 2$ & $1 / 3$ & 0.078 \\
$v_{r}$ & $1 / 2$ & 3 & 1 & 4 & 2 & 0.283 \\
$v_{i}$ & $1 / 2$ & 2 & $1 / 4$ & 1 & $1 / 4$ & 0.099 \\
$v_{t}$ & $1 / 2$ & 3 & $1 / 2$ & 4 & 1 & 0.215 \\
\hline \multicolumn{5}{c}{$\lambda_{\max }=5.338$} & $C R=0.075<0.10$ \\
\hline
\end{tabular}

Table 2 Comparison matrix and indicator weights After the selection of indicators, their standardization, and the definition of indicator weights, an analysis is performed in a raster environment using ArcGIS software to obtain the final PLMRI raster map. All weighted meteorological maps are overlaid using addition and the PLMRI raster map was then calculated using the Raster Calculator. Disregarding pixels with zero values or NODATA, the risk index values range from 0.10 to 0.58 (as shown in Figure 7). The closer these numbers are to 1 , the higher the likelihood of power line meteorological risk.

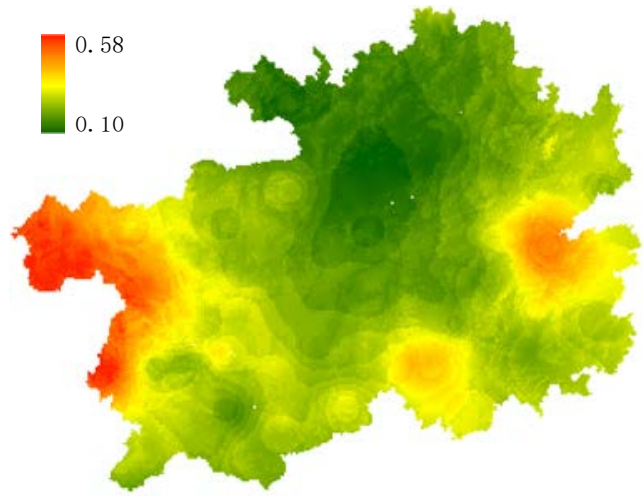

Figure 7 The PLMRI raster map

\subsection{The PLMRI value extraction}

Using Raster Calculator, the PLMRI raster map is generated which shows the spatial distribution of PLMRI values everywhere in Guizhou Province. But for power lines managers, the PLMRI value surrounding power lines is needed for meteorological risk early warning. This paper uses the buffer and overlay analysis to extract the PLMRI value surrounding power lines. Taking Tongli $220 \mathrm{kv}$ AC Line as an example, it is located in Qiandongnan Miao and Dong Autonomous Prefecture and passes through Yuping Dong Autonomous County, Zhenyuan County, Sansui County, Jinping County and Liping County from north to south.

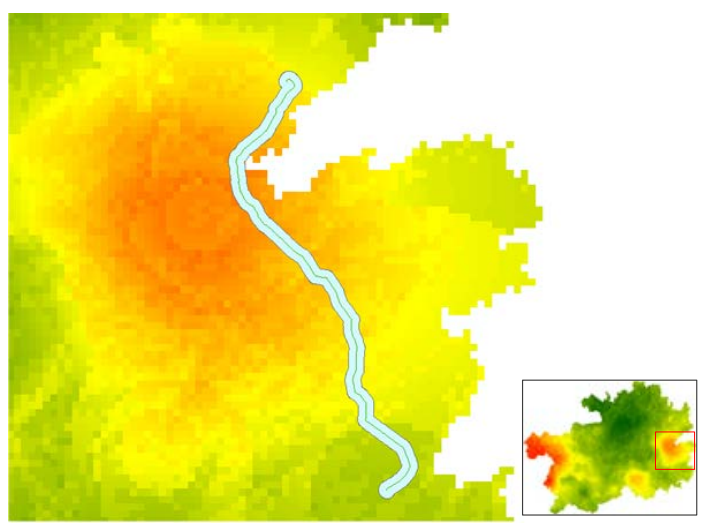

Figure 8 Buffer of Tongli Line on PLMRI raster map
Centred with every power line section of Tongli Line, buffers with radius of 0.02 radians are generated (as shown in Figure 8). The PLMRI value for each section of power lines can be extracted by finding the maximum value of PLMRI raster cell that is inside the power line section buffer. Figure 9 is a broken line graph that shows the PLMRI for each power line section of Tongli Line. From this graph, power line section with high meteorological risk can be intuitively seen, which provides an efficient way to help policymakers and power line manager to determine whether preventative maintenance is needed.

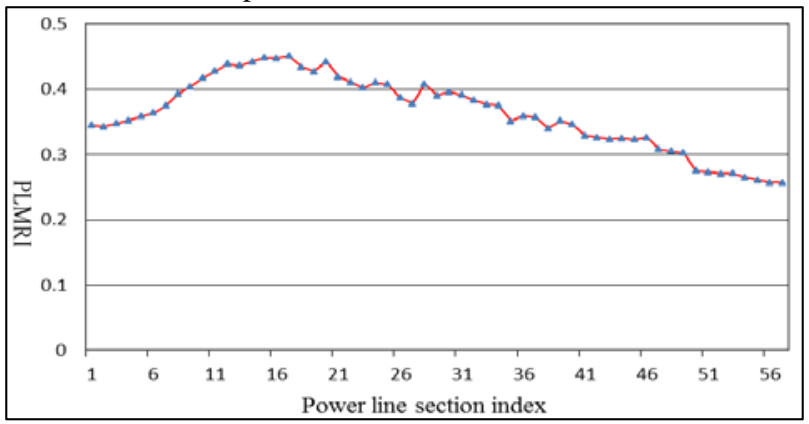

Figure 9 PLMRIs for each power line section

\subsection{Validation of PLMRI}

In order to validate the effectiveness of our proposed PLMRI model, this paper further conducts correlation analysis between the average PLMRI value and the corresponding cumulative failure times of each power lines according to the meteorological data of 2015 and operational data of 225 power lines. To rule out the influence of non- meteorological factors, we only count in the failures associated with meteorological factors, including wire breakdown, lightning failure, tower deformation, tower dumping and trip out caused by wind and ice. The results are shown in Table 3. Due to space limitations, only part of the data is selected.

\begin{tabular}{cccc}
\hline Index & Name & $\begin{array}{c}\text { Failure } \\
\text { Times }\end{array}$ & $\begin{array}{c}\text { Average } \\
\text { PLMRI }\end{array}$ \\
\hline 3654 & LanLiu Schort I & 20 & 0.41 \\
3651 & QiuKai Schort I & 26 & 0.66 \\
3640 & YongYang Schort II & 21 & 0.50 \\
3638 & XiangMa Schort & 19 & 0.25 \\
3617 & MeZhen Schort & 30 & 0.73 \\
3616 & AnYao Schort & 25 & 0.45 \\
3615 & ShiJiu Schort I & 21 & 0.44 \\
3614 & KaiXhuan Schort II & 26 & 0.50 \\
3613 & KaiXhuan Schort I & 13 & 0.19 \\
$\ldots . .$. & $\ldots . . .$. & $\ldots . .$. & $\ldots .$. \\
\hline
\end{tabular}

Table 3 Failure times and historical average PLMRI

Using the least squares method based linear model, we fit the historical average PLMRI value with failure times. The results are shown in Figure 10, in which $\mathrm{Y}$-axis is for historical average PLMRI value and $\mathrm{X}$-axis for total failure times. The fitting equation is $\mathrm{y}=0.0289 \mathrm{x}-0.1745$ with a determination coefficient of 0.8669 , which shows that there is a very good correlation between average PLMRI and the number of power line failure. So the PLMRI has certain explanatory power for power line failures, it also suggests that the meteorological risk evaluation model proposed in this paper is practical. 


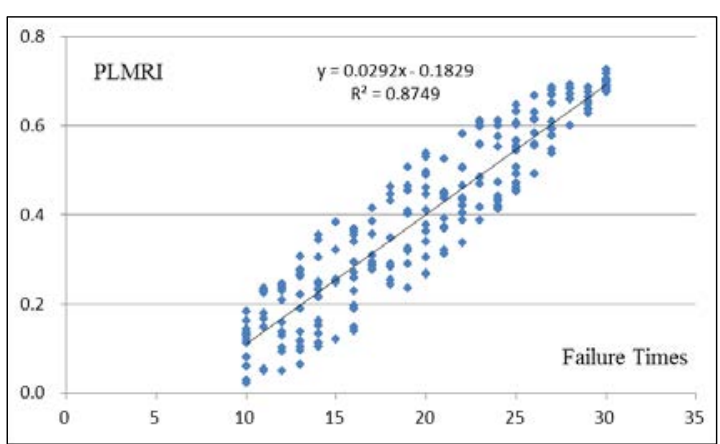

Figure 10 Correlation between PLMRI and failure times

\subsection{Management options}

The method developed for meteorological risk assessment for power lines can assist in the implementation of power line management strategies to prevent power line faults caused by meteorological factors. Specifically, the developed meteorological risk map for power lines can be useful: (a) for the early warning and protection of power lines against meteorological factors with a potential capacity for damage and (b) as an important tool for policy makers and managers from power administrative departments.

The local authorities should take reasonable protective measures (power line inspection, parts replacement, tower reinforcement, etc.) necessary to prevent potential meteorological damage for power lines in the high risk areas. Large areas in Guizhou Province can be classified as low or very low risk areas; these areas could consequently be preferable for future power line planning project as they are not prone to meteorological damage.

\section{CONCLUSIONS}

This paper puts forward a meteorological risk assessment method for power lines based on GIS and multi-sensor integration technology. In this research, multiple sensors were used to collect multiple meteorological data and analytic hierarchy process was performed to achieve the power line meteorological risk modelling. Finally, using GIS raster calculator, buffer analysis and overlay analysis, the meteorological risk for each power line section was calculated and extracted. This method provides a new and effective thought for the power line operation maintenance and meteorological risk management.

Qualitative risk assessment methods for depend on expert opinions. Being subjective, the results of these methods vary based on the knowledge of experts. By introducing analytic hierarchy process into meteorological risk assessment model, different experiences and knowledge of power line experts can be incorporated and then transferred into measurable meteorological risk weights, which make it possible for quantitative analysis of meteorological risk assessment. The assessment results of our proposed method will also be more reliable and efficient than that of a qualitative method.

Compared with traditional manual inspection and single sensor inspection, multi-sensor integration technology in the power line meteorological risk management has two obvious advantages: First, multi-sensors widely distributed in space make it possible for spatial interpolation of meteorological point data, which ensures the acquisition of meteorological value in wide areas and the meteorological risk assessment of any power lines in any position. Second, the meteorological risk model considering five kinds of multiple sensors can implement the comprehensive evaluation of power line meteorological risk, which makes up for the deficiency of the risk assessment method that considers only one meteorological factor.

This study also has room for improvement. For example, spatial interpolation method for meteorological factors can be chosen more scientifically according to the nature of different meteorological factors. Further research will introduce spatialtemporal interpolation method for meteorological point data to approximate the PLMRI value of any time.

\section{ACKNOWLEDGEMENTS}

This research was funded by the National Natural Science Foundation of China (Grant No.41271449, Grant No.41171350 and Grant No.61172175) and the Open Research Fund Program (No.13I01) of State Key Laboratory of Information Engineering in Surveying, Mapping and Remote Sensing (Wuhan University).

\section{REFERENCES}

Abdel-Gawad, A.F. and Zoklot, A.S.A., 2006. Wind and environmental effects on overhead high voltage transmission lines. In: U. Mander, C.A. Brebbia and E. Tiezzi (U. Mander, C.A. Brebbia and E. Tiezzi)^(U. Mander, C.A. Brebbia and E. Tiezzis)|,*WIT Transactions on Ecology and the Environment, pp. 433-443.

Aggarwal, R.K., Johns, A.T., Jayasinghe, J.A.S.B. and Su, W., 2000. An overview of the condition monitoring of overhead lines. Electric Power Systems Research, 53(1): 15-22.

Bagli, S., Geneletti, D. and Orsi, F., 2011. Routeing of power lines through least-cost path analysis and multicriteria evaluation to minimise environmental impacts. Environmental Impact Assessment Review, 31(3): 234-239.

Bai, H.F. and Li, H.N., 2009. Dynamic response of overhead transmission lines to oscillation caused by wind or rainfall loads. Power system technology, 33(2): 36-40.

Davidson, R. and Lambert, K., 2001. Comparing the Hurricane Disaster Risk of U.S. Coastal Counties. Natural Hazards Review, 2(3): 132-142.

Farzaneh, M. and Savadjiev, K., 2005. Statistical analysis of field data for precipitation icing accretion on overhead power lines. IEEE TRANSACTIONS ON POWER DELIVERY, 20(21): 1080-1087.

Haihong, C., Jing, Z. and Shuting, W., 2013. Development and Application of Electric Power Communication Line Management System Based on GIS. Electric Power System and its Automation(02): 26-30+64.

Ishizaka, A. and Labib, A., 2011. Review of the main developments in the analytic hierarchy process. EXPERT SYSTEMS WITH APPLICATIONS, 38(11): 14336-14345.

Jakše, J., Harash, A., Thabet, M. and McClure, G., 2001. Numerical modelling of snow-shedding effects on a $110 \mathrm{kV}$ overhead power line in Slovenia. The Eleventh International Offshore and Polar Engineering Conference. International 
Society of Offshore and Polar Engineers, 2001.

Jianli, J., Zhendong, Z., Zhi, L. and Gencheng, Q., 2003. Application of GIS in electric power communication network. Telecommunications for Electric Power System(06): 4-6+13.

Kikuchi, N., Matsuzaki, Y., Yukino, T. and Ishida, H., 2003. Aerodynamic drag of new-design electric power wire in a heavy rainfall and wind. Journal of wind engineering and industrial aerodynamics, 91(1): 41-51.

Liu, X., Zhang, J., Tong, Z. and Bao, Y., 2012. GIS-based multi-dimensional risk assessment of the grassland fire in northern China. NATURAL HAZARDS, 64(1): 381-395.

Qi, H. and Altinakar, M.S., 2011. A GIS-based decision support system for integrated flood management under uncertainty with two dimensional numerical simulations. Environmental Modelling \& Software, 26(6): 817-821.

Savadjiev, K. and Farzaneh, M., 2004. Modeling of icing and ice shedding on overhead power lines based on statistical analysis of meteorological data. Power Delivery, IEEE Transactions on, 19(2): 715-721.

Schoenherr, T., Rao Tummala, V.M. and Harrison, T.P., 2008. Assessing supply chain risks with the analytic hierarchy process: Providing decision support for the offshoring decision by a US manufacturing company. Journal of Purchasing and Supply Management, 14(2): 100-111.

Tang, A. and Wen, A., 2009. An intelligent simulation system for earthquake disaster assessment. Computers \& Geosciences, 35(5): 871-879.

Thompson, P.G. and Pilgrim, T., 2000. Apparatus and method of powerline surveying, designing in $3 \mathrm{D}$ and maintenance. Google Patents.

Tobler, W.R., 1970. A computer movie simulating urban growth in the Detroit region. Economic geography: 234-240.

Wang, Y., Li, Z., Tang, Z. and Zeng, G., 2011. A GIS-Based Spatial Multi-Criteria Approach for Flood Risk Assessment in the Dongting Lake Region, Hunan, Central China. WATER RESOURCES MANAGEMENT, 25(13): 3465-3484.

Yang, Y., Liu, Y. and Cao, Y., 2010. Study on a method of design for rural power distribution lines based on 3D GIS technology. Mathematical and Computer Modelling, 51(1112): 1293-1298.

Zerger, A. and Smith, D.I., 2003. Impediments to using GIS for real-time disaster decision support. Computers, environment and urban systems, 27(2): 123-141. 